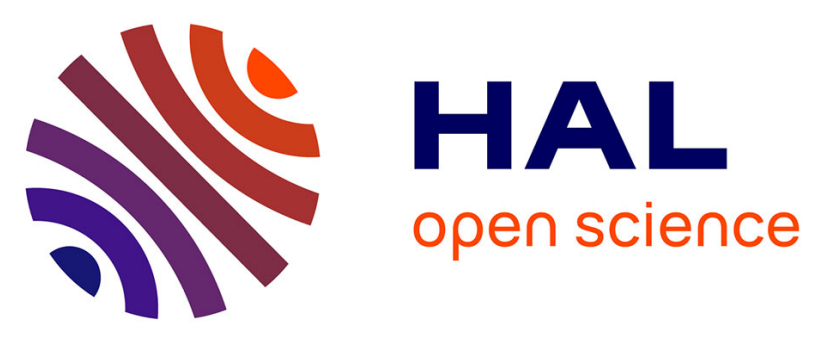

\title{
LQR-Mapped Fuzzy Controller Applied to Attitude Stabilization of a Power-Aided-Unicycle
}

\author{
Ping-Ho Chen, Wei-Hsiu Hsu, Ding-Shinan Fong
}

\section{To cite this version:}

Ping-Ho Chen, Wei-Hsiu Hsu, Ding-Shinan Fong. LQR-Mapped Fuzzy Controller Applied to Attitude Stabilization of a Power-Aided-Unicycle. 12th Engineering Applications of Neural Networks (EANN 2011) and 7th Artificial Intelligence Applications and Innovations (AIAI), Sep 2011, Corfu, Greece. pp.98-103, 10.1007/978-3-642-23960-1_12 . hal-01571467

\section{HAL Id: hal-01571467 \\ https://hal.inria.fr/hal-01571467}

Submitted on 2 Aug 2017

HAL is a multi-disciplinary open access archive for the deposit and dissemination of scientific research documents, whether they are published or not. The documents may come from teaching and research institutions in France or abroad, or from public or private research centers.
L'archive ouverte pluridisciplinaire HAL, est destinée au dépôt et à la diffusion de documents scientifiques de niveau recherche, publiés ou non, émanant des établissements d'enseignement et de recherche français ou étrangers, des laboratoires publics ou privés. 


\title{
LQR-Mapped Fuzzy Controller Applied to Attitude Stabilization of a Power-Aided-Unicycle
}

\author{
Ping-Ho Chen ${ }^{1}$, Wei-Hsiu Hsu ${ }^{2}$, Ding-Shinan Fong ${ }^{3}$ \\ ${ }^{123}$ Department of Electrical Engineering, Tungnan University, Taiwan \\ phc@mail.tnu.edu.tw
}

\begin{abstract}
Analysis of attitude stabilization of a power-aided unicycle points out that a unicycle behaves like an inverted pendulum subject to power constraint. An LQR-mapped fuzzy controller is introduced to solve this nonlinear issue by mapping LQR control reversely through least square and Sugeno-type fuzzy inference. The fuzzy rule surface after mapping remains optimal.
\end{abstract}

Keywords: LQR, Fuzzy, Inverted pendulum, Unicycle, Stabilization.

\section{Introduction}

This paper introduces the control of unicycle stabilization using an inversed optimal fuzzy controller [1][2][3] mapped from a LQR controller. Since control of unicycle stabilization is constrained by power and torque limitation due to motor specification, engineers will meet difficulty in implementing LQR [4][5][6] control surface owing to its nonlinearity. Dynamics of unicycle stabilization [7] and fuzzy transformation from LQR, will be dealt with in depth equivalently in this paper.

The goal of unicycle stabilization is to minimize attitude and rate of attitude of the rider, or equivalently the seat, by rider's mechanical power and fuzzy electrical power simultaneously. Proposed in this paper is an EM (electrical/mechanical) unicycle that has mass center right above the top of the wheel as if it were an inverted pendulum referred to Fig. 1(a)-(b). Since the seat is no longer as high as the traditional one, shortening of torque arm reduces manual torque and accordingly deteriorates maneuverability. With the help of auxiliary electrical power, it would be beneficial to compensate for torque reduction and furthermore to increase the stabilization effects.

Fig. 1(a)-(b) shows the seat plane (above wheel axle) and pedal platform (underneath wheel axle). Mass $M$ (with arm $L$ ) and mass $m$ (with arm $l$ ) represent masses distributed above and underneath the wheel axle respectively. The total mass center $M+m$ located at $l_{p}$ above the axle behaves as if it were an inverted pendulum. The pendulum arm is

$$
l_{p}=(M L-m l) / m_{p}, L=l_{s}+l_{r}, m_{p}=M+m
$$


where $l_{s}$ :length between seat and wheel; $l_{r}$ : length between mass center of rider's body and seat. Fig. 1(c) shows riding on the move.
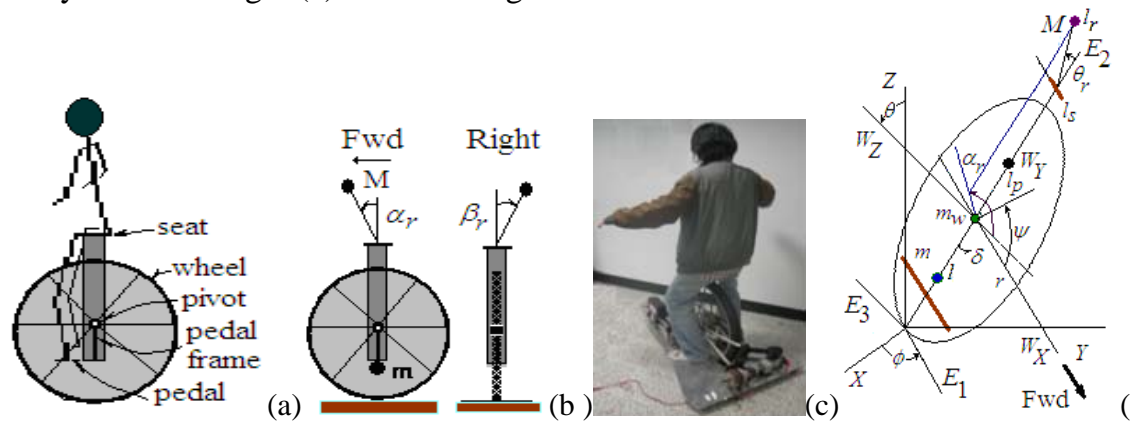

Fig. 1. (a) Schematic diagram of EM unicycle. (b) Pendulum mass $M$ and $m$ viewed from side and back. (c) Rider driving on the move. (d) Geometry of unicycle.

Control of the unicycle combines rider's manual controller and a complementary fuzzy controller. Manual torques $\boldsymbol{\tau}_{\boldsymbol{m}}$ and fuzzy torque $\boldsymbol{\tau}_{f}$ are outputs of both controllers defined by $\tau_{m}:\left[\tau_{m P} \tau_{m R}\right]^{\mathrm{t}}, \tau_{m R}:$ roll torque, $\tau_{m P}:$ pitch torque, $\boldsymbol{\tau}_{f}:\left[\tau_{f w} \tau_{f P} \tau_{f R}\right]^{\mathrm{t}}$ (fuzzy torque), $\tau_{f w}$ : fuzzy wheel torque, $\tau_{f P}$ : fuzzy pitch torque, $\tau_{f R}$ : fuzzy roll torque.

\section{Dynamics of Unicycle}

There are five coordinates involved in the dynamics, i.e. inertia coordinate $[X, Y, Z]^{t}$ $\rightarrow$ Euler coordinate $\left[E_{1}, E_{2}, E_{3}\right]^{t} \rightarrow$ wheel coordinate $\left[W_{X}, W_{Y}, W_{Z}\right]^{t} \rightarrow$ pendulum coordinate $\left[P_{1}, P_{2}, P_{3}\right]^{t} \rightarrow$ rider coordinate $\left[R_{1}, R_{2}, R_{3}\right]^{t}$. Note that Euler coordinate $\left[E_{1}, E_{2}, E_{3}\right]^{t}$ is transformed from inertia coordinate $[X, Y, Z]^{t}$ by turning an angle of $\phi$ along axis $Z$ and then tilting an angle of $\theta$ along axis $E_{1}$.

Application of Newton's second law [7] in matrix form gives

$$
\begin{aligned}
& \dot{\boldsymbol{H}}+\boldsymbol{\omega} \boldsymbol{H}=\boldsymbol{\tau}, \quad \boldsymbol{\omega}=\left[\begin{array}{ccc}
0 & -\omega_{3} & \omega_{2} \\
\omega_{3} & 0 & -\omega_{1} \\
-\omega_{2} & \omega_{1} & 0
\end{array}\right], \boldsymbol{H}=\left[h_{1} h_{2} h_{3}\right]^{t}=\left[I_{1} \Omega_{1}, I_{2} \Omega_{2}, I_{3} \Omega_{3}\right]^{t} \\
& I_{1}=I_{w x}+m_{w} r^{2}+I_{p 1}+m_{p}\left(r+l_{p}\right)^{2} I_{2}=I_{w y}+I_{p 2} I_{3}=I_{w z}+m_{w} r^{2}+I_{p 3}+m_{p}\left(r+l_{p}\right)^{2}
\end{aligned}
$$

For $i=1,2,3, \boldsymbol{H}$ : angular momentum of unicycle; $I_{i}$ : moment of inertia of unicycle along Euler axis $E_{i} ; I_{w x}$ : moment of inertia of wheel along wheel $\operatorname{axis} W_{x} ; m_{w}$ : wheel mass; $r$ : wheel radius; $I_{p i}$ :moment of inertia of total pendulum along pendulum axis $P_{1} ; \Omega_{i}$ :inertia angular rate along Euler axis $E_{i} ; \omega$ :precession matrix; $\boldsymbol{\tau}$ :applied torque in Euler coordinate; $\psi$ : wheel rotation; $\alpha_{r}$ : rider's pitch angle along 
rider axis $R_{3} ; \theta_{r}$ :rider's roll tilt angle along rider axis $R_{1} ; \delta$ :platform pitch angle; $l$ : length between mass center of lower pendulum and wheel axle; $g$ :gravity. Further derivation yields

$$
\begin{gathered}
I_{1} \dot{\Omega}_{1}-I_{2} \omega_{3} \Omega_{2}+I_{3} \omega_{2} \Omega_{3}=\tau_{1}=\tau_{v 1}+\tau_{m R}+\tau_{f R} \\
\tau_{v 1}=\tau_{u} \cos \theta, \tau_{u}=-\left[m_{w} r+m_{p}\left(r+l_{p}\right)\right] g \\
I_{2} \dot{\Omega}_{2}-I_{3} \omega_{1} \Omega_{3}+I_{1} \omega_{3} \Omega_{1}=\tau_{v 2}, \tau_{v 2}=\tau_{u} \sin \theta \\
I_{3} \dot{\Omega}_{3}-I_{1} \omega_{2} \Omega_{1}+I_{2} \omega_{1} \Omega_{2}=\tau_{f W}
\end{gathered}
$$

Where $\tau_{v 1}$ and $\tau_{v 2}$ comes from gravity effect accompanied with wheel tilt. Eq. (4), (5) and (6) correspond respectively to the dynamics of roll, heading and wheel drive.

\section{Stabilization of Pitch Based on LQR}

Stabilization of pitch based on LQR depends on the analysis of pitch-wheel coupling. Governing equations, based on force and torque are:

$$
\begin{gathered}
\left(m_{w}+m_{p}\right) \ddot{x}+b \dot{x}-m_{p} l_{p} \ddot{\delta} \cos \delta+m_{p} l_{p} \dot{\delta}^{2} \sin \delta=\frac{\tau_{f W}}{r} \\
\left(I_{p 3}+m_{p} l_{p}^{2}\right) \ddot{\delta}-m_{p} l_{p} \ddot{x} \cos \delta=m_{p} g l_{p} \sin \delta+\tau_{m P}+\tau_{f P}
\end{gathered}
$$

Where $I_{p 3}$ : moment of inertia of inverted pendulum along mass center located at $l_{p}$ above the axle. While stabilized, $\delta \approx 0$ and $\dot{\delta} \approx 0$, Eq. (7) and (8) are represented in matrix form as

$$
\left[\begin{array}{c}
\ddot{x} \\
\dot{\delta} \\
\ddot{\delta}
\end{array}\right]=\left[\begin{array}{ccc}
-b / \hat{m} & g m_{p}{ }^{2} l_{p}{ }^{2} / \hat{m} \bar{I} & 0 \\
0 & 0 & 1 \\
-m_{p} l_{p} b /(\bar{m} \hat{I}) & m_{p} g l_{p} / \hat{I} & 0
\end{array}\right]\left[\begin{array}{l}
\dot{x} \\
\delta \\
\dot{\delta}
\end{array}\right]+\left[\begin{array}{cc}
1 / \hat{m} r & m_{p} l_{p} / \hat{m} \bar{I} \\
0 & 0 \\
m_{p} l_{p} / \bar{m} \hat{I} r & 1 / \hat{I}
\end{array}\right]\left[\begin{array}{c}
\tau_{f W} \\
\tau_{m P}+\tau_{f P}
\end{array}\right]
$$

Where $\hat{I}=\bar{I}-m_{p}{ }^{2} l_{p}{ }^{2} / \bar{m}, \hat{m}=\bar{m}-m_{p}{ }^{2} l_{p}{ }^{2} / \bar{I} \quad, \quad \bar{m}=m_{w}+m_{p}, \bar{I}=I_{p 3}+m_{p} l_{p}{ }^{2}$ Obviously, three eigen values of transition matrix in Eq. (9) are $\lambda_{1}<0, \lambda_{2}=0$, $\lambda_{3}>0$. This results in an unstable pendulum system in the presence of drive-pitch coupling. Eq. (9) can be expressed by

$$
\dot{\boldsymbol{X}}_{1}=\boldsymbol{A}_{1} \boldsymbol{X}_{1}+\boldsymbol{B}_{1} \boldsymbol{u}_{1}, \quad \boldsymbol{Y}_{1}=\boldsymbol{C}_{1} \boldsymbol{X}_{1} \text { (Subscript } 1 \text { for pitch and } 2 \text { for roll) }
$$

In Eq. (10), $\boldsymbol{X}_{1}, \boldsymbol{u}_{1}$ and $\boldsymbol{Y}_{1}$ are state, control and measurement respectively. Assigning $\quad M=60 \mathrm{~kg}, m=15 \mathrm{~kg}, m_{w}=10 \mathrm{~kg}, r=0.52 \mathrm{~m}, b=0.5 \mathrm{nt} / \mathrm{m} / \mathrm{s}, l=0.2 \mathrm{~m}$, 
$l_{s}=0.18 \mathrm{~m}, g=9.8 \mathrm{~m} / \mathrm{s}^{2}$, it follows $m_{p}=75 \mathrm{~kg}, l_{p}=0.52 \mathrm{~m}, I_{p 3}=9.7 \mathrm{~kg}-\mathrm{m}^{2}$, $\bar{I}=30 \mathrm{~kg}-\mathrm{m}^{2}, \quad \bar{m}=85 \mathrm{~kg}, \hat{m}=24.3 \mathrm{~kg}, \hat{I}=12.1 \mathrm{~kg}-\mathrm{m}^{2}$.

Eq. (9) becomes

$$
\boldsymbol{X}_{1}=\left[\begin{array}{c}
\dot{x} \\
\delta \\
\dot{\delta}
\end{array}\right], \quad \boldsymbol{u}_{1}=\left[\begin{array}{c}
\tau_{f W} \\
\tau_{m P}+\tau_{f P}
\end{array}\right], \boldsymbol{A}_{1}=\left[\begin{array}{ccc}
-0.02 & 50.69 & 0 \\
0 & 0 & 1 \\
-0.02 & 31.59 & 0
\end{array}\right], \boldsymbol{B}_{1}=\left[\begin{array}{cc}
.079 & .133 \\
0 & 0 \\
.073 & .083
\end{array}\right], \boldsymbol{C}_{1}=\boldsymbol{I}_{3 \times 3}
$$

Since Eigen values of $\boldsymbol{A}_{1}$ are $0.0121,-5.6365,5.6044$, it implies the original system of unicycle is unstable. If control torque $\boldsymbol{u}_{1}$ is substituted by state feedback - $\boldsymbol{K}_{1} \boldsymbol{X}_{1}$ with $\boldsymbol{Q}_{1}=\boldsymbol{I}_{3 \times 3}, \boldsymbol{R}_{1}=\boldsymbol{I}_{2 \times 2}$, the optimal gain $\boldsymbol{K}_{1}$ will be

$$
\boldsymbol{K}_{1}=\left[\begin{array}{rrr}
-0.4144 & 377.1436 & 67.5795 \\
1.0834 & 431.9048 & 74.9096
\end{array}\right]
$$

Eigen values of the LQR are $-0.04,-5.73$ and -5.52 that guarantee the system to be stable.

\section{Stabilization of Roll Based on LQR}

Substituting $\Omega_{1}=\omega_{1}=\dot{\theta}, \quad \Omega_{2}=\omega_{2}=\dot{\phi} \sin \theta, \Omega_{3}=\omega_{3}+\dot{\psi}=\dot{\phi} \cos \theta+\dot{\psi} \quad$ and $\dot{\psi}=$ constant, $\dot{\phi}=$ constant, $\theta=\alpha+\pi / 2, \alpha$ : roll angle ( $\alpha \approx 0$ ) into Eq. (4), it follows

$$
I_{1} \ddot{\alpha}+k \alpha=\tau_{h}+\tau_{m R}+\tau_{f R}, k=\tau_{u}-\left(I_{3}-I_{2}\right) \dot{\phi}^{2}, \tau_{h}=-I_{3} \dot{\psi} \dot{\phi}
$$

The term $\tau_{h}$ is a reaction torque caused by precession of angular momentum. Assigning that wheel speed is set at $5 \mathrm{~km} / \mathrm{hr}$ (i.e. $1.39 \mathrm{~m} / \mathrm{s}$ ), then making a quarter turn needs $11.31 \mathrm{sec}$. It yields $\dot{\phi}=0.139 \mathrm{rad} / \mathrm{s}$ and $\dot{\psi}=2.67 \mathrm{rad} / \mathrm{s}$. Else parameters are: $I_{1}=95.5 \mathrm{~kg}-\mathrm{m}^{2}, I_{2}=6.15 \mathrm{~kg}-\mathrm{m}^{2}, I_{3}=94.8 \mathrm{~kg}-\mathrm{m}^{2}, \tau_{u}=815.4 \mathrm{nt}-\mathrm{m}, k=813.7 \mathrm{nt}-\mathrm{m}$.

$$
\boldsymbol{X}_{2}=\left[\begin{array}{c}
\alpha \\
\dot{\alpha}
\end{array}\right], \quad \boldsymbol{u}_{2}=\tau_{\boldsymbol{m} R}+\tau_{f R}, \boldsymbol{A}_{2}=\left[\begin{array}{cc}
0 & 1 \\
-119.7 & 0
\end{array}\right], \boldsymbol{B}_{2}=\left[\begin{array}{c}
0 \\
0.01
\end{array}\right], \boldsymbol{C}_{2}=\boldsymbol{I}_{2 \times 2}
$$

Since eigen values of $\boldsymbol{A}_{2}$ are $10.9407 i$ and -10.9407i, it implies the original system of unicycle is critical stable. If control torque $\boldsymbol{u}_{2}$ is substituted by state feedback - $\boldsymbol{K}_{2} \boldsymbol{X}_{2}$ with $\boldsymbol{Q}_{2}=500 \boldsymbol{I}_{2 \times 2}, \boldsymbol{R}_{2}=0.01$, the optimal gain $\boldsymbol{K}_{2}$ will be

$$
\boldsymbol{K}_{2}=\left[\begin{array}{ll}
2.1 & 225
\end{array}\right]
$$

Eigen values of the LQR are $-1.1227+10.8840 \mathrm{i}$ and $-1.1227-10.8840 \mathrm{i}$ that guarantees the system to be stable. 


\section{Inversed Fuzzy Controller Mapped from LQR [8]}

Taking roll stabilization as an instance, the LQR control is $u_{2}=225 \dot{\alpha}+2.1 \alpha$ constrained by

$$
P_{2}=\tau_{2} \dot{\alpha}=\boldsymbol{u}_{2} \dot{\alpha}=\tau_{f R} \dot{\alpha}=\left(\boldsymbol{K}_{2} \boldsymbol{X}_{2}\right) \dot{\alpha}=\left(k_{21} \alpha+k_{22} \dot{\alpha}\right) \dot{\alpha} \leq P_{2 \max }
$$

For $P_{2 \max }=100, \quad \boldsymbol{u}_{2} \dot{\alpha} \leq 100$ (Inequality constraint)

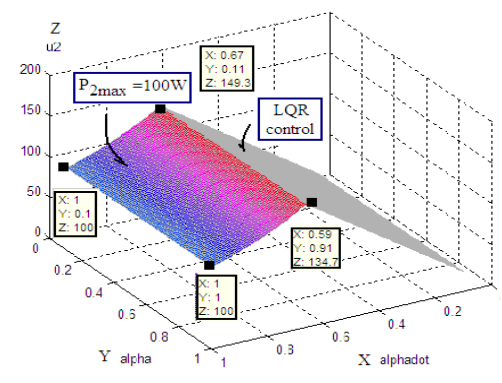

(a)

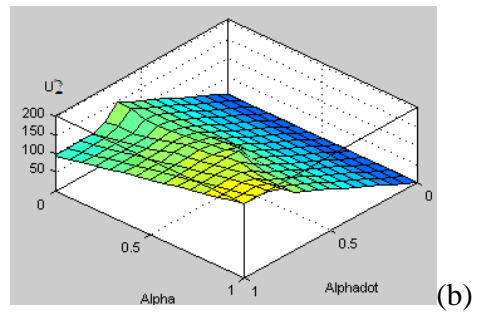

Fig. 2. (a) LQR control with power constraint (b) Inversed fuzzy controller after mapping Preprocessing data assumption: 1) $\alpha$ and $\dot{\alpha}$, as fuzzy variables are normalized, i.e. $\alpha=1 \mathrm{rad}$ and $\dot{\alpha}=1 \mathrm{rad} / \mathrm{s} \quad 2$ ) Maximum power $P_{2 \max }$ is $100 \mathrm{w}$, i.e. $\tau_{2 \max }=100 \mathrm{nt}-\mathrm{m}$ at $\dot{\alpha}=1 \mathrm{rad} / \mathrm{s}$. 3) Apex of hyperboloid surface due to power constraint is indicated in Fig. 2.(a). 4) Plane $p_{1}$ (LQR) $u_{2}=225 \dot{\alpha}+2.1 \alpha$ is also shown in Fig. 2.(a). 5) Plane $p_{2}$, simplified from hyperboloid surface by least square [8], is:

$$
u_{2}=\boldsymbol{\Lambda}\left[\begin{array}{lll}
\dot{\alpha} & \alpha & 1
\end{array}\right]^{\mathrm{T}}, \boldsymbol{\Lambda}=\left[\begin{array}{lll}
-108.3 & -12.3 & 216.1
\end{array}\right]
$$

The fuzzy rule surface, as a minimum of $p_{1}$ and $p_{2}$, is able to be implemented by reversely finding fuzzy membership and inference using Sugeno model [9] as follows.
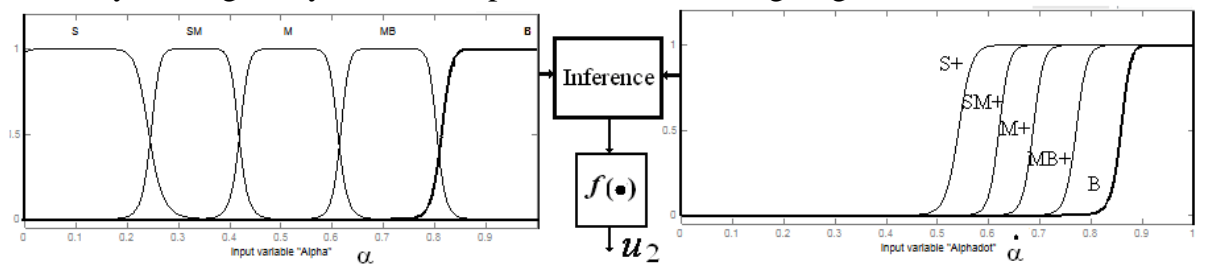

Fig. 3. Membership function of $\alpha$ and $\dot{\alpha}$

Membership functions of fuzzy variables $\alpha$ and $\dot{\alpha}$ are given in Fig. 3 that also shows Sugeno FIS (Fuzzy inference system) [8]. Sugeno linear models is employed in the inference as given below:

1. If ( $\alpha$ is S) and ( $\dot{\alpha}$ is B) then ( $u$ is $p$ ); $\quad$ 2. If ( $\alpha$ is SM) and ( $\dot{\alpha}$ is $\mathrm{MB}+$ ) then ( $u$ is $p 2)$

3. If ( $\alpha$ is $\mathrm{M}$ ) and ( $\dot{\alpha}$ is $\mathrm{M}+$ ) then ( $u$ is $p 2$ ); 4 . If ( $\alpha$ is MB) and ( $\dot{\alpha}$ is $\mathrm{SM}+$ ) then ( $u$ is $p 2)$

5. If ( $\alpha$ is B) and ( $\dot{\alpha}$ is S+) then ( $u$ is $p$ ); $\quad 6$. If ( $\alpha$ is S) and ( $\dot{\alpha}$ is not B) then ( $u$ is $p 1$ ) 
7. If ( $\alpha$ is $\mathrm{SM}$ ) and ( $\dot{\alpha}$ is not $\mathrm{MB}+$ ) then ( $u$ is $p 1$ ); 8. If ( $\alpha$ is $\mathrm{M}$ ) and ( $\dot{\alpha}$ is not $\mathrm{M}+$ ) then ( $u$ is $p 1$ ); 9. If ( $\alpha$ is $\mathrm{MB}$ ) and ( $\dot{\alpha}$ is not $\mathrm{SM}+$ ) then ( $u$ is $p 1$ ); 10 . If ( $\alpha$ is B) and ( $\dot{\alpha}$ is not $\mathrm{S}+$ ) then $(u$ is $p 1)$.

Where linear surfaces $p_{1}=225 \dot{\alpha}+2.1 \alpha$ and $p_{2}=-108.3 \dot{\alpha}-12.3 \alpha+216.1$

The fuzzy rule surface, thus obtained as given in Fig. 2.(b), is very close to LQR control surface in Fig. 2.(a). Therefore the inversed fuzzy controller mapped from LQR remains optimal although it needs more massage. Fig. 2 and Fig. 3 are plotted by using Matlab fuzzy toolbox [10].

\section{Conclusion}

Through the analysis of unicycle dynamics, we find that stabilization control of a power-aided unicycle, in pitch and roll, meets difficulty in using LQR control under power and torque constraints. This paper applies the approach, called "inversed fuzzy controller mapped from LQR controller” to solve the issue of stabilization control of unicycle. A Sugeno fuzzy control surface, almost equivalent LQR control surface, is built by using fuzzy membership and inference. Through this kind of transformation, we have the equivalent control surface, still remaining optimal, but using fuzzy-logiccontroller (FLC) to implement.

\section{References}

1. Zadeh, L.A.,: The concept of a linguistic variable and its application to approximate reasoning, Parts 1, 2, and 3. Information Sciences, 1975, 8:199-249, 8:301-357, 9:43-80.

2. Mamdani, E. H. and Assilian S., : An Experiment in Linguistic Synthesis with Fuzzy Logic Controller. Int. J. Man Mach. Studies, 7, No.1, pp1-13, 1975.

3. Mamdani, E.H., : Advances in the linguistic synthesis of fuzzy controllers. International Journal of Man-Machine Studies, Vol. 8, pp. 669-678, 1976.

4. Zadeh, L. A., : A Theory of Approximating Reasoning. Reading: Elsevier, pp.149-194, 1979.

5. Frank L. Lewis, : Applied Optimal Control \& Estimation. Prentice Hall, 1992.

6. Jeffrey B. Burl,: Linear Optimal Control. Addison-Wesley, 1999.

7. Charles W. Bert, : Dynamics and stability of unicycles and monocycles. Dynamical Systems, 1468-9375, Volume 5, Issue 1, 1990.

8. Ping-Ho Chen, Kuang-Yow Lian, : Optimal Fuzzy Controller Mapped from LQR under Power and Torque Constraints. 7th AIAI Conferences, 2011.

9. Sugeno, M., : Fuzzy measures and fuzzy integrals: a survey. (M.M. Gupta, G. N. Saridis, and B.R. Gaines, editors), Fuzzy Automata and Decision Processes, pp. 89-102, North-Holland, NY,1977.

10. Matlab: toolbox:Fuzzy, 2010 ed.. 